\title{
Human Factors Operability Timeline Analysis to Improve the Processing Flow of the Orion Spacecraft
}

\author{
Roland Schlierf \& Damon B. Stambolian \\ NASA Kennedy Space Center (KSC) \\ Constellation Ground Operations Project \\ JFK Space Center \\ KSC, FL 32899 \\ roland.schlierf-1@nasa.gov \& damon.b.stambolian@nasa.gov \\ List of co-authors: Ms. Darcy Miller, NASA KSC; Mr. Juan Posada, BOE KSC; \\ Mr. Mike Haddock, NASA KSC; Mr. Mike Haddad, NASA KSC; Mr. Donald Tran, NASA KSC; Dr. Gena Henderson, \\ NASA KSC; and Dr. Tim Barth, NASA Engineering and Safety Center (NESC)
}

\begin{abstract}
The Constellation Program (CxP) Orion vehicle goes through several areas and stages of processing before its launched at the Kennedy Space Center. In order to have efficient and effective processing, all of the activities need to be analyzed. This was accomplished by first developing a timeline of events that included each activity, and then each activity was analyzed by operability experts and human factors experts with spacecraft processing experience. This papers focus is to explain the results and the process for developing this human factors operability timeline analysis to improve the processing flow of Orion. ${ }^{12}$
\end{abstract}

\section{TABLE OF CONTENTS}

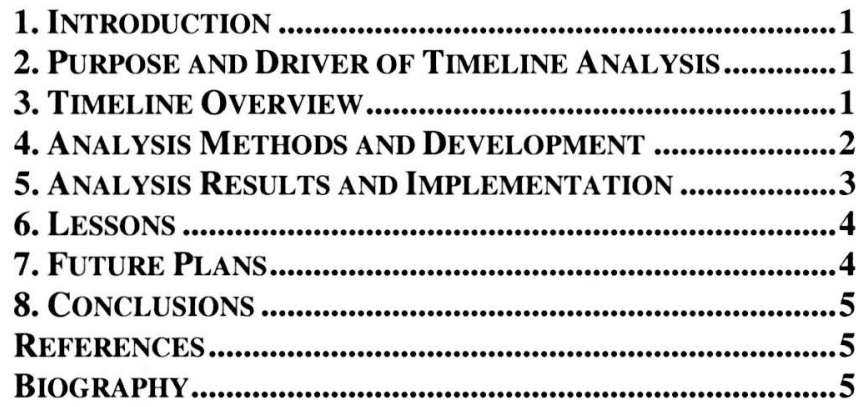

\section{INTRODUCTION}

The Constellation Program (CxP) Orion vehicle goes through several areas and stages of processing before its launched at the Kennedy Space Center. During all of the stages of this flow the ground processes were analyzed. This was accomplished by first developing a timeline of events that included each activity, and then each activity was analyzed by operability experts and human factors experts with spacecraft processing experience. A human factors analysis tool was adapted for this activity. Several human factors, and operations experts were brought together for a small team to perform this analysis. Following each issue discovered during the analysis the necessary analysis design engineering was brought into the process in order to address the issue for design improvements.

\section{Purpose and Driver of Timeline Analysis}

The purpose of the timeline analysis was to look at each step of the ground processing of the Orion vehicle as it goes through the different stages of processing at KSC, with an human factors and operations eye on the hardware human interactions affecting the human performance during assembly, maintenance, inspection of Orion at KSC.

When an issue was discovered in the timeline, applicable standards were married with the issue. These standards mainly came from the FAA Human Factors Design Standards (HFDS). This process was basically stripping out the applicable FAA requirements that apply to ground processing of Orion, given the operations methodologies specially derived by the GOP to meet the $\mathrm{CxP}$ directives for $\mathrm{CxP}$. The requirements derived during this exercise may or may not be applicable to another vehicle. If the processes are different during a different timeline, then another analysis would need to be performed.

\section{Timeline OVERVIEW}

The Orion processing time line includes Delivery from the Orion assembly line (located at KSC), Orion Processing in Multi Purpose Processing Facility, Orion/Ares Integration and Testing in the Vehicle Integration Building (VAB), Pad Operations, Landing and Recovery and, Post Flight processing.

For each location a functional flow block diagram was developed. An example of the Functional Flow Block Diagram (FFBD) for the VAB is shown in figure 1 . 


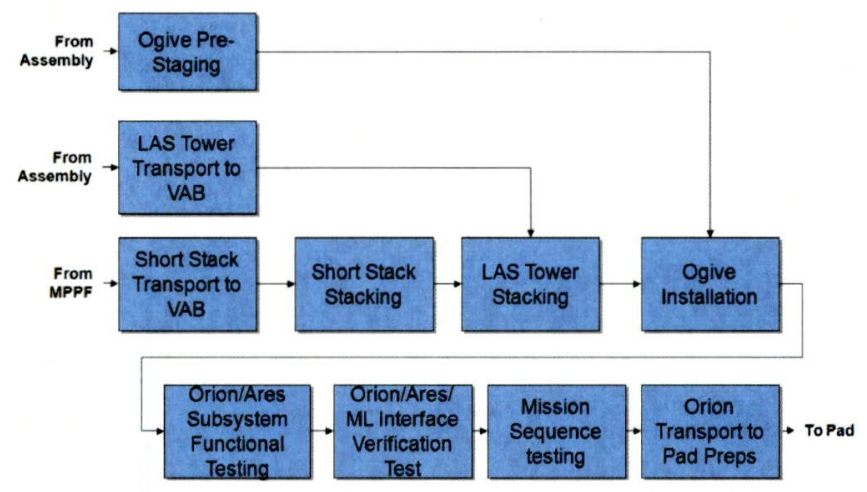

With each of these high level areas, there are lower tiers of FFBDs. In those lower level FFBDs there are several tasks that take place. These tasks/activity are defined in the Orion Processing Flow during the Human Factors Operability Engineering Analysis (HFOEA). Each of those tasks were given an operations human factors engineering analysis.

This human factors analysis included qualified human factors engineers, operations engineers and design engineers and the design visualization team. Prior to meeting with the human factors engineers, design visualation of the flight and ground hardware and humans were developed using the design visualation process. The visualization process is where the operations folks meet with the design visualization folks in the design visualization lab and reviewed the flight and GSE designs for human factors. These important meetings with the operations and design

\section{ANAlysis Methods AND DEVElopment}

The analysis was basically a step by step processing going though each operations step to complete a task which would complete a block. Once one block was completed the next block was analyzed. The team modified a Human Factors Engineering Analysis (HFEA) tool developed by the KSC Engineering Directorate for the CxP GOP subsystems HFEA [2], basically by re-arranging the analysis spreadsheet to show a timeline of events. For each of the events the 5 areas in the tool were addressed. There are 5 major areas of the tool. They are; Human Interfaces, Issue, Processing Phase, Risk Analysis, and Recommendations. The first area is the human interface. This area includes; Location of process, Human/System Interface (H/SI), Human Interface, Task, FFBD Event Number, Task/Issues/Actions (TIA), and Conditions (The italicized were additions to the original HFEE Tool, these are shown in yellow in Figure 1.) The second area identifies the requirements to address the issues; the Requirement Source (RS) (typically FAA), the Section Title, (RST) Subsection Title (RSST), Requirements Verbiage (RV), if the requirement is satisfied or not (RS), and the primary verification method (PVM), which is Analysis, Inspection, Demo, or test. The third area included the processing phase (PP); Assembly/Installation, Nominal Use, Inspection, Maintenance, Off-Nominal Use, Emergency Use and Disassembly/ Disposal. The fourth section included the risk analysis section, Possible Consequences (PC), Priority Rank Likelihood (PRL),

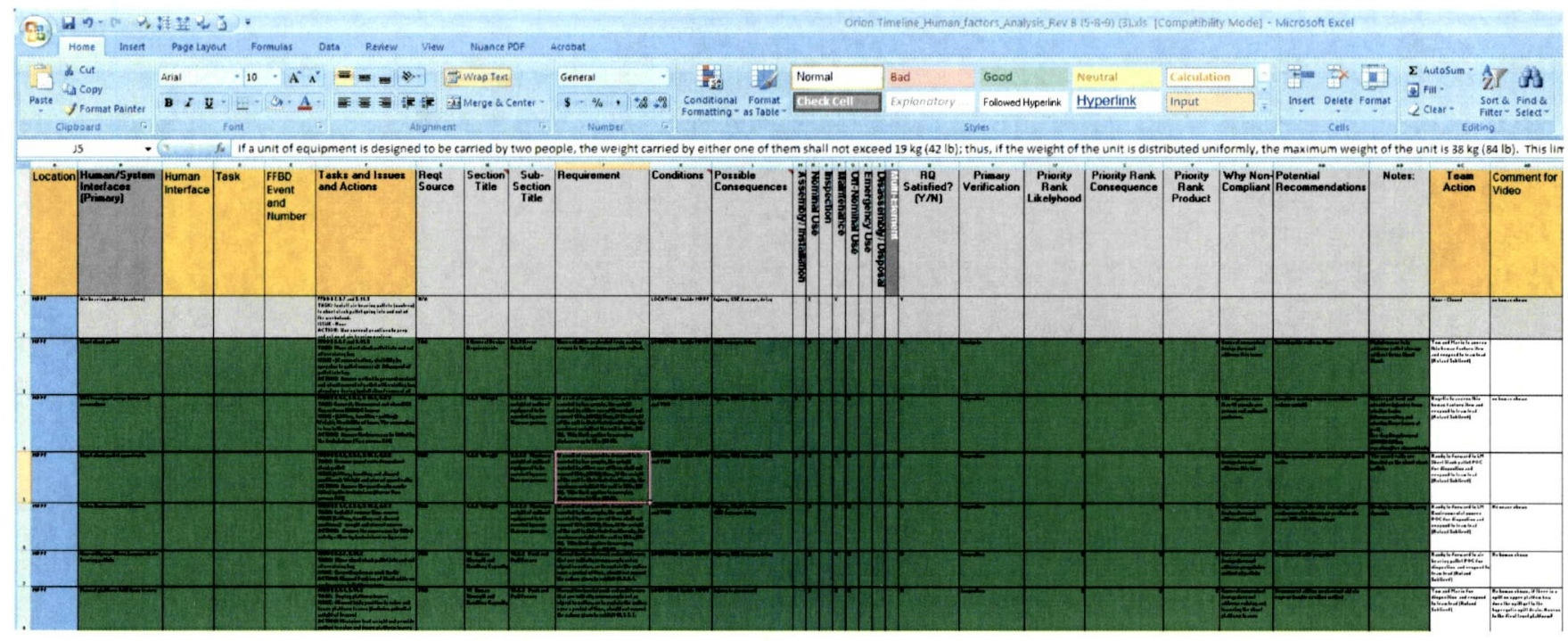

Figure 1 Modified HFEA Tool for Orion Processing Flow during the Human Factors Operability Engineering Analysis

(HFOEA)

engineers using the actual flight and ground designs loaded into the design visualization tools were a great help during the Human Factors Operability Engineering Analysis (HFOEA).
Priority Rank Consequence (PRC), Priority Rank Product (PRP), and if currently none compliant the reason why (NCR). The fifth area provided; Potential Recommendations (PR), Notes (N), Team Action (TA), and comments for human factor video/animation improvements $(\mathrm{CV})$. All of these areas are shown within the first row of the Modified 
HFEA Tool, Figure 1. The grayed out row $\# 2$ is a closed issue, the rows in green are in work.

\section{EXAMPLES FROM THE ANALYSIS}

Several human factors issues from figure-1 that will be explained here as examples. Because the information in the spreadsheet is too vast to fit into this paper, each cell will be explained in these examples.

For Example \#1 in the spreadsheet, the task of moving the short stack pallet into and out of servicing bay was evaluated. Alignment of pallet into the servicing bay was considered an issue that required further evaluation. An action was taken to assure a method is put in place to prevent contact and misalignment of pallet with existing bay structure during installation/removal of short stack pallet ". A human factors requirement was evaluated and applied to the task that states "Users shall be protected from making errors to the maximum possible extent". A recommendation was provided to the design team to install guide rails on floor. The spreadsheet fields were populated as follows:

- H/SI, - Short stack pallet.

- TIAs -

- TASK: Move short stack pallet into and out of servicing bay.

- ISSUE - (Communication, visibility by operator to pallet corners): Alignment of pallet into bay.

- ACTION - Assure method to prevent contact and misalignment of pallet with existing bay structure during installation/removal of short stack pallet ".

- $\quad$ RS - FAA.

- $\quad$ RST - 2-General Design Requirements.

- RSST - 2.5.3 Error Resistant.

- $\quad \mathrm{RV}$ - "Users shall be protected from making errors to the maximum possible extent".

- PC - GSE damage, delay.

- PP - Nominal Use and Maintenance.

- PVM - Analysis.

- $\quad$ PL - 2 .

- PRC - 2 .

- PRP - 4.

- NCR - Conceptual design does not address this issue.

- PR- Install guide rails on floor.

- Notes - Maintenance is to address pallet storage without Orion Short Stack.

- TA - Team Members to assess this human factors item and respond to team lead.

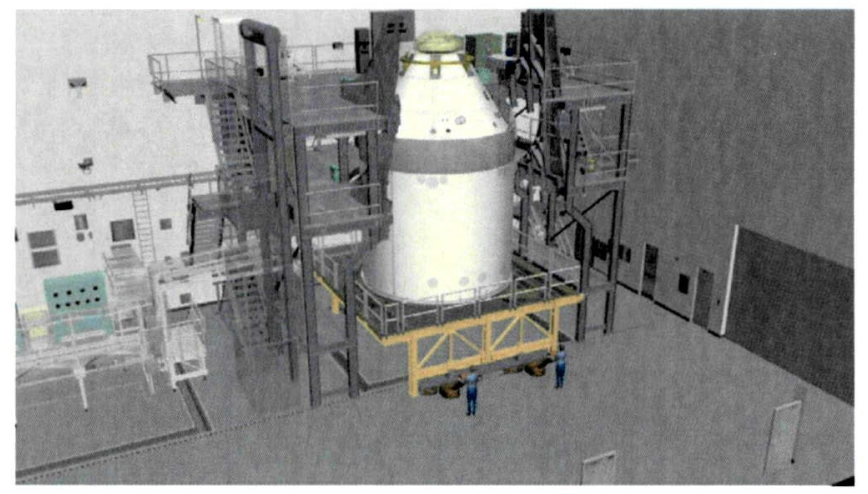

For Example \#2 in the spreadsheet, the task of connecting, disconnecting, and stowing hoses from transporter was evaluated. The weight and flexibility of the hoses was considered an issue. An action was taken by the team to assure the hoses can be lifted by the technicians. Human factors requirements for maximum weight of units of equipment to be carried by more than one person were assessed. If a unit of equipment is designed to be carried by two people, the weight carried by either one of them shall not exceed $19 \mathrm{~kg}(42 \mathrm{lb})$; thus, if the weight of the unit is distributed uniformly, the maximum weight of the unit is 38 $\mathrm{kg}(84 \mathrm{lb})$. This limit applies to carrying distances up to 10 $\mathrm{m}$ (33 ft). It was recommended to change the design of the hoses to be in sections to reduce weight to below these human factors requirements. The spreadsheet fields were populated as follows:

- H/SI - ECS transport purge hoses and connectors.

- TIAs -

o TASK: Connect, Disconnect and stow ECS Hoses from KAMAG Source.

○ ISSUE - (Lifting, handling - pulling): Weight, flexibility of hose. The connection is low to the ground.

- ACTION: Assure the hoses can be lifted by the technicians (Two person lift).

- RS - FAA.

- RST - 4.2.2 Weight.

- RSST - 4.2.2.6 Maximum weight of units of equipment to be carried by more than one person.

- RV - "If a unit of equipment is designed to be carried by two people, the weight carried by either one of them shall not exceed $19 \mathrm{~kg}(42 \mathrm{lb})$; thus, if the weight of the unit is distributed uniformly, the maximum weight of the unit is $38 \mathrm{~kg}(84 \mathrm{lb})$. This limit applies to carrying distances up to $10 \mathrm{~m}$ (33 ft). Injury, GSE damage, delay."

- PC - Injury, GSE damage, delay

- PPs - Nominal Use and Maintenance. Inspection.

- $\quad$ PL - 3.

- $\quad$ PRC - 2 .

- PRP - 6 . 
- NCR - the lift requires more than 44 pounds per person and awkward postures.

- $\quad$ PR - Consider making hoses in sections to reduce weight. The notes were, "History of back and shoulder injuries from similar tasks (disconnecting and storing these hoses at pad). See Gap Requirement (NIOSH lifting equation)for akward body positions.

- TA - Rogelio to assess this human factors item and respond to team lead. The $\mathrm{CV}$ was that there was no human shown.

For Example \#3 in the spreadsheet, the task of removing guard rails from short stack pallet was also addressed. It had a similar issue to the hoses described above. The issue was for the weight and size of guard rails. An action was taken by the team to assure the guard rails can be lifted by the technicians. Similar human factors requirements applied. It was recommended that the design ensure manageable size and weight of the guard rails that meets the requirements. The spreadsheet fields were populated as follows:

- H/SI - Short stack pallet guard rails.

- The TIAs are

o TASK: Remove guard rails from short stack pallet.

- ISSUE (Lifting, handling and akward ,positions): Weight and size of guard rails.

- ACTION: Assure the guard rails can be lifted by the technicians (One or Two person lift).

- RS - FAA.

- $\mathrm{RS}$ - 4.2.2.

- RST - 4.22 Weight.

- RSST - 4.2.2.6 Maximum weight of units of equipment to be carried by more than one person

- RV - "If a unit of equipment is designed to be carried by two people, the weight carried by either one of them shall not exceed $19 \mathrm{~kg}(42 \mathrm{lb})$; thus, if the weight of the unit is distributed uniformly, the maximum weight of the unit is $38 \mathrm{~kg}(84 \mathrm{lb})$. This limit applies to carrying distances up to $10 \mathrm{~m}$ (33 $\mathrm{ft}$ ).

- $\quad$ PC - Injury, GSE damage, delay.

- PPs - Assembly/Installation, Nominal Use, and OffNominal Use.

- PVM - Inspection.

- $\quad$ PRL -3.

- PRC - 2 .

- PRP - 6.

- NCR - current conceptual design does not address this issue.

- PR - Design manageable size and weight guard rails. The notes are that the guard rails are located on the short stack pallet.
- TA - Randy to forward to LM Short Stack pallet POC for disposition and respond to team lead.

\section{LESSONS}

This section is a collection of lessons learned that will be documented into the NASA Integrated Lessons Learned system. http://nen.nasa.gov/portal/site/lis/LL/ Lessons learned include both positive lessons as well as lessons for making improvements.

(1) The NASA CxP program level human factors requirements document HSIR greatly promoted better human factors Systems Engineering and Integration. This improved the integration between ground systems, crewed vehicle designs for ground processing [4].

(2) Early collaboration and planning between the flight and ground hardware designers for human factors operability engineering analysis (HFEA) is necessary.

(3) Timeline analysis is great way to analyze and improve the design of ground and flight hardware interfaces for ground processing of the ground equipment, and the flight and ground hardware interface-

(4) Although the HFOEA was primarily applied to Orion and GS to improve the design of hardware for better human factors and operations, some areas of HFOEA can be used to improve the work operations documents and processes. Example, for hazard warnings signs being called out in the work steps, etc.

(5) Employ qualified human factors person/s on team from the beginning of the Project.

(6) Human factors engineers should perform the human factors assessments as embedded members of the HFOEA design teams.

\section{Future Plans}

(1) Continue applying these processes and collaborations to future NASA missions in the $21^{\text {st }}$ Century. Specifically the timeline option to the Human Factors Engineering Analysis Tool (HFEAT) where applicable.

(2) Promote more design integration processes and designs between KSC, MSFC, and JSC, by using the timeline option for human factors operations engineering analysis.

(3) Promote sharing of this process across Centers, projects, and with commercial partners.

(4) Embed HFEA and HFOEA as part of the Engineering processes, as a decision tools to choose from for the appropriate human factors engineering analysis. 
(5) Continue using the design visualization process, where operations met with the design visualization folks in the design visualization lab and reviewed the flight and GSE designs for human factors. These important meetings with the operations and design engineers using the actual flight and ground designs loaded into the design visualization tools were a great help.

(6) Introduce motion capture analysis into applicable activities during the human factors operability engineering timeline analysis, especially where a worksite analysis is needed or where two or more projects interface, Ares/Orion/GO [3]. See Figure-2 Motion capture allows for quicker and simpler and real to life simulations, and the computer models will include the CAD flight hardware and human Avatar which the envelope spaces between the human and flight hardware can be viewed, and the stresses to the human can be computed. See reference [3] for how motion capture was used during development of Orion for ground operations. See reference [4] for more on the CxP level 2 requirements that promoted better design of flight hardware for ground processing.

(7) Promote more HFEOA timeline analysis with the ground support equipment, such as the flight and ground interface at the umbilical plates and the ground commodity connections to flight hardware.

(8) Use the design problems and solutions from this HFEOA effort to improve the NASA STD 3001, to promote better NASA human factors Systems Engineering and Integration in future programs.

(9) Employ the human factors systems engineering processes and lessons learned from Orion to future launch vehicles.

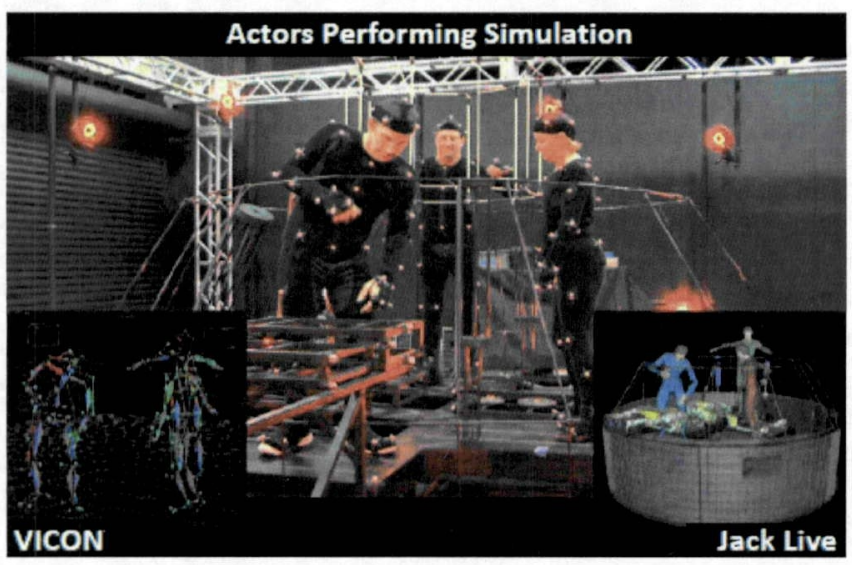

Figure 2 Motion Capture of Operations inside the Orion vehicle.

\section{Conclusions}

This paper described the successful modification and application of the HFEA Tool for a timeline human factors operability engineering analysis for the Orion vehicle. Through this and other human interface analysis there was great progress made during $\mathrm{CxP}$ with collaboration between the Orion Project, Ground Operations Project and KSC Design Engineering. The most difficult and time consuming aspects of the development of these teams and analysis during $\mathrm{CxP}$ were during the forming, storming and norming stages, to get to the performing stages where we are today. Now that this has been accomplished, these methods and collaborations for human factors operability engineering analysis will continue to improve and carry over as we lead the nation in developing spacecraft systems for future missions.

\section{REFERENCES}

[1] Damon B. Stambolian, Scott Wilson, Kirk Logsdon, Darcy Miller, Jihan Dinally, Jason, Masse, and Tim Barth. "1-G Human Factors for Optimal Processing and Operability of Constellation Ground Systems" 2009 IEEEACpaper\#1286

[2] Damon B. Stambolian, Gena Henderson, Darcy Miller, Donald Tran. "1-G Human Factors for Optimal Processing and Operability of Ground Systems up to CxP PDR" 2011 IEEEACpaper\#xxxx

[3] Jeffrey S. Osterlund \& Brad A. Lawrence. 61st Virtual Reality: Avatars in Human Spaceflight Training. International Astronautical Congress, Prague, CZ. Copyright $\odot 2010$ by the International Astronautical Federation.

http://kave.ksc.nasa.gov/HEMAP/HEMAP2010/HEMAP2 010.mp4

[4] Dischinger, Charles H. Jr. and Stambolian Damon B. and Miller Darcy H. "The first development of human factors engineering requirements for application to ground task design for a NASA flight program" Aerospace SAE Publications 2008-01-2103. http://papers.sae.org/2008-01$\underline{2103}$

\section{BIOGRAPHY}

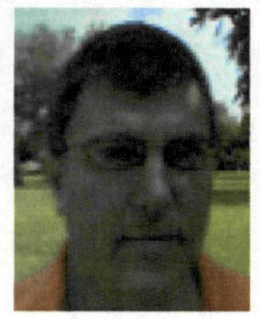

Damon Stambolian is currently working on a PhD in Industrial Engineering focusing his research on Biomechanics at the University of Miami's Biomechanics Laboratory. He is also currently working in the KSC Engineering Directorate at Kennedy Space Center. Prior to working in the 
Engineering Directorate he worked in; the Constellation Ground Operations Project office, the Space Station Program within the Orbiter Space Plane Project at KSC, and the Space Shuttle Program at KSC. Within these Programs he was involved with human factors related process improvements for ground processing operations, i.e., assembly, maintenance, inspection of flight hardware.

List of acknowledgements for others that had influenced the development of the CXP GOP human factors at KSC; Jeffrey Angermeier, Kelvin Manning, Gary Letchworth, Doug Nelson, Brad Lawrence, Rogelio Franco, Randy Eastman, Tom Miller and Mario Relvini, Jason Hopkins, Chris Thompson, Jeanie Ruiz, Matt Craycraft, Gary Richards, Bob Ruiz, and Blake Hale. 